ELECTRONIC RESEARCH ANNOUNCEMENTS OF THE AMERICAN MATHEMATICAL SOCIETY

Volume 13, Pages 33-45 (April 12, 2007)

S $1079-6762(07) 00172-2$

\title{
q-EULERIAN POLYNOMIALS: EXCEDANCE NUMBER AND MAJOR INDEX
}

\author{
JOHN SHARESHIAN AND MICHELLE L. WACHS
}

(Communicated by Sergei Fomin)

\begin{abstract}
In this research announcement we present a new $q$-analog of a classical formula for the exponential generating function of the Eulerian polynomials. The Eulerian polynomials enumerate permutations according to their number of descents or their number of excedances. Our $q$-Eulerian polynomials are the enumerators for the joint distribution of the excedance statistic and the major index. There is a vast literature on $q$-Eulerian polynomials that involves other combinations of Eulerian and Mahonian permutation statistics, but this is the first result to address the combination of excedance number and major index. We use symmetric function theory to prove our formula. In particular, we prove a symmetric function version of our formula, which involves an intriguing new class of symmetric functions. We also discuss connections with (1) the representation of the symmetric group on the homology of a poset introduced by Björner and Welker; (2) the representation of the symmetric group on the cohomology of the toric variety associated with the Coxeter complex of the symmetric group, studied by Procesi, Stanley, Stembridge, Dolgachev, and Lunts; (3) the enumeration of words with no adjacent repeats studied by Carlitz, Scoville, and Vaughan and by Dollhopf, Goulden, and Greene; and (4) Stanley's chromatic symmetric functions.
\end{abstract}

\section{INTRODUCTION}

The subject of permutation statistics originated in the early 20th century work of Major Percy MacMahon [22, Vol. I, pp. 135, 186; Vol. II, p. viii], 23], and it has developed into an active and important area of enumerative combinatorics over the last four decades. It deals with the enumeration of permutations according to natural statistics. A permutation statistic is simply a function from the symmetric group $\mathfrak{S}_{n}$ to the set of nonnegative integers. MacMahon studied four fundamental permutation statistics, the inversion index, the major index, the descent number, and the excedance number, which we define below.

Let $[n]$ denote the set $\{1,2, \ldots, n\}$. For each $\sigma \in \mathfrak{S}_{n}$, the descent set of $\sigma$ is defined to be

$$
\operatorname{DES}(\sigma):=\{i \in[n-1]: \sigma(i)>\sigma(i+1)\}
$$

Received by the editors October 16, 2006.

2000 Mathematics Subject Classification. Primary 05A30, 05E05, 05E25.

The first author was supported in part by NSF Grants DMS 0300483 and DMS 0604233, and the Mittag-Leffler Institute.

The second author was supported in part by NSF Grants DMS 0302310 and DMS 0604562 , and the Mittag-Leffler Institute.

(C)2007 American Mathematical Society Reverts to public domain 28 years from publication 
and the excedance set is defined to be

$$
\operatorname{EXC}(\sigma):=\{i \in[n-1]: \sigma(i)>i\} .
$$

The descent number and excedance number are defined respectively by

$$
\operatorname{des}(\sigma):=|\operatorname{DES}(\sigma)| \quad \text { and } \quad \operatorname{exc}(\sigma):=|\operatorname{EXC}(\sigma)| .
$$

For example, if $\sigma=32541$, written in one line notation, then

$$
\operatorname{DES}(\sigma)=\{1,3,4\} \quad \text { and } \quad \operatorname{EXC}(\sigma)=\{1,3\} ;
$$

hence $\operatorname{des}(\sigma)=3$ and $\operatorname{exc}(\sigma)=2$. If $i \in \operatorname{DES}(\sigma)$ we say that $\sigma$ has a descent at $i$. If $i \in \operatorname{EXC}(\sigma)$ we say that $\sigma(i)$ is an excedance of $\sigma$.

MacMahon [22, Vol. I, p. 186] observed that the descent number and excedance number are equidistributed, that is, the number of permutations in $\mathfrak{S}_{n}$ with $j$ descents equals the number of permutations with $j$ excedances for all $j$. (There is a well-known combinatorial proof of this fact due to Foata [11, 14.) These numbers were first studied by Euler and have come to be known as the Eulerian numbers. They are the coefficients of the Eulerian polynomial 1

$$
A_{n}(t):=\sum_{\sigma \in \mathfrak{S}_{n}} t^{\operatorname{des}(\sigma)}=\sum_{\sigma \in \mathfrak{S}_{n}} t^{\operatorname{exc}(\sigma)} .
$$

Any permutation statistic that is equidistributed with des and exc is said to be an Eulerian statistic.

The Eulerian numbers and the Eulerian polynomials have been extensively studied in many different contexts in the mathematics and computer science literature. For excellent treatments of this subject, see the classic lecture notes of Foata and Schützenberger [15], and Section 5.1 of Knuth's classic book series "The Art of Computer Programming" 21]. The exponential generating function formula,

$$
\sum_{n \geq 0} A_{n}(t) \frac{z^{n}}{n !}=\frac{1-t}{e^{z(t-1)}-t}
$$

where $A_{0}(t)=1$, is attributed to Euler in [21, p. 39].

The major index of a permutation $\sigma \in \mathfrak{S}_{n}$ is defined by

$$
\operatorname{maj}(\sigma):=\sum_{i \in \operatorname{DES}(\sigma)} i
$$

and the inversion statistic is defined by

$$
\operatorname{inv}(\sigma):=|\{(i, j): 1 \leq i<j \leq n \& \sigma(i)>\sigma(j)\}| .
$$

MacMahon 23] showed that the major index is equidistributed with the inversion statistic by establishing the first equality in

$$
\sum_{\sigma \in \mathfrak{S}_{n}} q^{\operatorname{maj}(\sigma)}=[n]_{q} !=\sum_{\sigma \in \mathfrak{S}_{n}} q^{\operatorname{inv}(\sigma)},
$$

where

$$
[n]_{q}:=1+q+\cdots+q^{n-1}
$$

and

$$
[n]_{q} !:=[n]_{q}[n-1]_{q} \cdots[1]_{q} .
$$

Rodrigues 28 had earlier obtained the second equality. (An elegant combinatorial proof of the equidistribution of maj and inv was obtained by Foata [12, 14.) Any

\footnotetext{
${ }^{1}$ It is more common to define the Eulerian polynomials as $\sum_{\sigma \in \mathfrak{S}_{n}} t^{\operatorname{des}(\sigma)+1}$.
} 
permutation statistic that is equidistributed with the major index and inversion index is said to be a Mahonian statistic.

Note that by setting $q=1$ in (1.2), one gets the formula $n$ ! for the number of permutations. Equation (1.2) is a beautiful " $q$-analog" of this formula and is the fundamental example of the subject of permutation statistics and $q$-analogs, in which one seeks to obtain nice $q$-analogs of enumeration formulas.

One can look for nice $q$-analogs of the Eulerian polynomials by considering the joint distributions of the Mahonian and Eulerian statistics given above. Consider the four possibilities,

$$
\begin{aligned}
A_{n}^{\mathrm{inv}, \operatorname{des}}(q, t) & :=\sum_{\sigma \in \mathfrak{S}_{n}} q^{\operatorname{inv}(\sigma)} t^{\operatorname{des}(\sigma)} \\
A_{n}^{\operatorname{maj}, \operatorname{des}}(q, t) & :=\sum_{\sigma \in \mathfrak{S}_{n}} q^{\operatorname{maj}(\sigma)} t^{\operatorname{des}(\sigma)} \\
A_{n}^{\mathrm{inv}, \operatorname{exc}}(q, t) & :=\sum_{\sigma \in \mathfrak{S}_{n}} q^{\operatorname{inv}(\sigma)} t^{\operatorname{exc}(\sigma)} \\
A_{n}^{\text {maj,exc }}(q, t) & :=\sum_{\sigma \in \mathfrak{S}_{n}} q^{\operatorname{maj}(\sigma)} t^{\operatorname{exc}(\sigma)}
\end{aligned}
$$

There are many interesting results on the first three $q$-Eulerian polynomials and on multivariate distributions of all sorts of combinations of Eulerian and Mahonian statistics (for a sample see [1, 2, 15, 17, 13, 16, 17, 18, 19, 20, 26, 27, 29, 30, 31, 38]). These include Stanley's 31] $q$-analog of (1.1), given by

$$
\sum_{n \geq 0} A_{n}^{\mathrm{inv}, \operatorname{des}}(q, t) \frac{z^{n}}{[n]_{q} !}=\frac{1-t}{\operatorname{Exp}_{q}(z(t-1))-t},
$$

where $A_{0}^{\text {inv,des }}(q, t)=1$ and

$$
\operatorname{Exp}_{q}(z):=\sum_{n \geq 0} q^{\left(\begin{array}{c}
n \\
2
\end{array}\right)} \frac{z^{n}}{[n]_{q} !} .
$$

Surprisingly, we have found no mention of the fourth $q$-Eulerian polynomial $A_{n}^{\text {maj,exc }}(q, t)$ anywhere in the literature. Here we announce the following remarkable $q$-analog of (1.11).

Theorem 1.1. The q-exponential generating function for $A_{n}^{\operatorname{maj}, \operatorname{exc}}(q, t)$ is given by

$$
\sum_{n \geq 0} A_{n}^{\text {maj,exc }}(q, t) \frac{z^{n}}{[n]_{q} !}=\frac{(1-t q) \exp _{q}(z)}{\exp _{q}(z t q)-t q \exp _{q}(z)},
$$

where $A_{0}^{\text {maj,exc }}(q, t)=1$ and

$$
\exp _{q}(z):=\sum_{n \geq 0} \frac{z^{n}}{[n]_{q} !} .
$$

An alternative formulation of (1.3) more closely analogous to (1.1) is given by

$$
\sum_{n \geq 0} A_{n}^{\text {maj,exc }}(q, t) \frac{z^{n}}{[n]_{q} !}=\frac{1-t q}{\exp _{q}(z t q) \operatorname{Exp}_{q}(-z)-t q} .
$$

In fact, we prove the following more general result, which reduces to Theorem 1.1 when $r=1$. 
Theorem 1.2. Let

$$
A_{n}^{\text {maj,exc,fix }}(q, t, r):=\sum_{\sigma \in \mathfrak{S}_{n}} q^{\operatorname{maj}(\sigma)} t^{\operatorname{exc}(\sigma)} r^{\mathrm{fix}(\sigma)},
$$

where $\operatorname{fix}(\sigma)$ denotes the number of fixed points of $\sigma \in \mathfrak{S}_{n}$, i.e., the number of $i \in[n]$ such that $\sigma(i)=i$. Then

$$
\sum_{n \geq 0} A_{n}^{\text {maj,exc,fix }}(q, t, r) \frac{z^{n}}{[n]_{q} !}=\frac{(1-t q) \exp _{q}(r z)}{\exp _{q}(z t q)-t q \exp _{q}(z)} .
$$

By setting $t=1$ in (1.5) one obtains a formula of Gessel and Reutenauer [19], and by setting $q=1$ one obtains a formula that is equivalent to (1.1). By setting $r=0$, we obtain the new result,

$$
\sum_{n \geq 0} \sum_{\sigma \in \mathcal{D}_{n}} q^{\operatorname{maj}(\sigma)} t^{\operatorname{exc}(\sigma)} \frac{z^{n}}{[n]_{q} !}=\frac{1-t q}{\exp _{q}(z t q)-t q \exp _{q}(z)},
$$

where $\mathcal{D}_{n}$ is the set of derangements in $\mathfrak{S}_{n}$.

Alternative formulations of (1.5) are given by the recurrence relation

$$
A_{n}^{\text {maj,exc,fix }}(q, t, r)=r^{n}+\sum_{k=0}^{n-2}\left[\begin{array}{l}
n \\
k
\end{array}\right]_{q} A_{k}^{\text {maj,exc,fix }}(q, t, r) t q[n-k-1]_{t q},
$$

and the formula

$$
A_{n}^{\text {maj,exc,fix }}(q, t, r)=\sum_{m=0}^{\left\lfloor\frac{n}{2}\right\rfloor}(t q)^{m} \sum_{\substack{k_{0} \geq 0 \\
k_{1}, \ldots, k_{m} \geq 2 \\
\sum k_{i}=n}}\left[\begin{array}{c}
n \\
k_{0}, \ldots, k_{m}
\end{array}\right]_{q} r^{k_{0}} \prod_{i=1}^{m}\left[k_{i}-1\right]_{t q},
$$

where

$$
\left[\begin{array}{c}
n \\
k
\end{array}\right]_{q}=\frac{[n]_{q} !}{[k]_{q} ![n-k]_{q} !} \text { and }\left[\begin{array}{c}
n \\
k_{0}, \ldots, k_{m}
\end{array}\right]_{q}=\frac{[n]_{q} !}{\left[k_{0}\right]_{q} !\left[k_{1}\right]_{q} ! \cdots\left[k_{m}\right]_{q} !} .
$$

In the next section we describe the techniques that were used to prove Theorem 1.2. They involve an interesting class of symmetric functions and a symmetric function identity which generalizes Theorem 1.2. We prove the symmetric function identity by modifying a bijection of Gessel and Reutenauer [19] and generalizing a bijection of Stembridge [35. After a preliminary version of this paper was circulated, Foata and Han extended Theorem 1.2, finding the generating function for the joint distribution of maj, exc, fix, and des. Their result can also be obtained by specializing our symmetric function identity.

In Section 3 we discuss a connection with two graded representations of the symmetric group, which turn out to be isomorphic. We show that a specialization of the Frobenius characteristic of these representations yields $A^{\text {maj,exc }}(q, t)$. One of the representations is the representation of the symmetric group on the cohomology of the toric variety associated with the Coxeter complex of the symmetric group. This representation was studied by Procesi [25], Stanley [32], Stembridge [35, 36, and Dolgachev and Lunts 9 . The other representation is the representation of the symmetric group on the homology of maximal intervals of a certain intriguing poset introduced by Björner and Welker [4] in their study of connections between poset topology and commutative algebra. In fact, our study of the latter representation is 
what led us to discovering formula (1.1) and its symmetric function generalization, in the first place.

Various authors have studied Mahonian (resp. Eulerian) partners to Eulerian (resp. Mahonian) statistics whose joint distribution is equal to a known EulerMahonian distribution. We mention, for example, Foata 13, Foata and Zeilberger [17, Clarke, Steingrímsson, and Zeng [7, Haglund [20, Babson and Steingrímsson 1], and Skandera 29]. In Section 4 we define a new Mahonian statistic to serve as a partner for des in the (maj, exc) distribution. We do not have a simple proof of the equidistribution. We have a highly nontrivial proof which uses tools from poset topology and the symmetric function results announced in Sections 2 and 3

Details of the proofs discussed in this announcement, as well as further consequences and open problems, will appear in a forthcoming paper.

\section{Symmetric FunCtion GenERALizATION}

In this section we present a symmetric function generalization of Theorem 1.2 , Let

$$
H(z)=H(\mathbf{x}, z):=\sum_{n \geq 0} h_{n}(\mathbf{x}) z^{n},
$$

where $h_{n}(\mathbf{x})$ denotes the complete homogeneous symmetric function in the indeterminates $\mathbf{x}=\left(x_{1}, x_{2}, \ldots\right)$, that is,

$$
h_{n}(\mathbf{x}):=\sum_{1 \leq i_{1} \leq i_{2} \leq \cdots \leq i_{n}} x_{i_{1}} x_{i_{2}} \cdots x_{i_{n}}
$$

for $n \geq 1$, and $h_{0}=1$. By setting $x_{i}:=q^{i-1}$, for all $i$, and $z:=z(1-q)$ in $H(\mathbf{x}, z)$, one obtains $\exp _{q}(z)$; see [33]. It follows that

$$
\left.\frac{(1-t) H(\mathbf{x}, z r)}{H(\mathbf{x}, z t)-t H(\mathbf{x}, z)}\right|_{\substack{x_{i}:=q^{i-1} \\ z:=z(1-q)}}=\frac{(1-t) \exp _{q}(z r)}{\exp _{q}(z t)-t \exp _{q}(z)} .
$$

We will construct, for each $n, j, k \geq 0$, a quasisymmetric function $Q_{n, j, k}(\mathbf{x})$ whose generating function $\sum_{n, j, k \geq 0} Q_{n, j, k}(\mathbf{x}) t^{j} r^{k} z^{n}$ specializes to

$$
\sum_{n \geq 0} \sum_{\sigma \in \mathfrak{S}_{n}} q^{\operatorname{maj}(\sigma)-\operatorname{exc}(\sigma)} t^{\operatorname{exc}(\sigma)} r^{\mathrm{fix}(\sigma)} \frac{z^{n}}{[n]_{q} !}
$$

when we set $x_{i}:=q^{i-1}$ and $z:=z(1-q)$. Thus, by taking specializations of both sides of (2.4) below and setting $t:=t q$, we obtain (1.5).

For $\sigma \in \mathfrak{S}_{n}$, let $\bar{\sigma}$ be the barred word obtained from $\sigma$ by placing a bar above each excedance. For example, if $\sigma=531462$ then $\bar{\sigma}=\overline{5} \overline{3} 14 \overline{6} 2$. View $\bar{\sigma}$ as a word over the ordered alphabet

$$
\{\overline{1}<\overline{2}<\cdots<\bar{n}<1<2<\cdots<n\} .
$$

We extend the definition of descent set from permutations to words $w$ of length $n$ over an ordered alphabet by letting

$$
\operatorname{DES}(w):=\left\{i \in[n-1]: w_{i}>w_{i+1}\right\},
$$

where $w_{i}$ is the $i$ th letter of $w$. Now define the excedance-descent set of a permutation $\sigma \in \mathfrak{S}_{n}$ to be

$$
\operatorname{EXD}(\sigma):=\operatorname{DES}(\bar{\sigma})
$$


For example, $\operatorname{EXD}(531462)=\operatorname{DES}(\overline{5} \overline{3} 14 \overline{6} 2)=\{1,4\}$. The interesting thing about EXD is that for all $\sigma \in \mathfrak{S}_{n}$,

$$
\sum_{i \in \operatorname{EXD}(\sigma)} i=\operatorname{maj}(\sigma)-\operatorname{exc}(\sigma)
$$

For $S \subseteq[n-1]$ and $n \geq 1$, define the quasisymmetric function

$$
F_{S, n}\left(x_{1}, x_{2}, \ldots\right):=\sum_{\substack{i_{1} \geq \cdots \geq i_{n} \\ j \in S \Rightarrow i_{j}>i_{j+1}}} x_{i_{1}} \cdots x_{i_{n}}
$$

and let $F_{\emptyset, 0}=1$. A basic result in Gessel's theory of quasisymmetric functions (see [33. Lemma 7.19.10]) is that

$$
F_{S, n}\left(1, q, q^{2}, \ldots\right)=\frac{q^{\sum_{s \in S} s}}{(1-q)\left(1-q^{2}\right) \cdots\left(1-q^{n}\right)} .
$$

Hence it follows from (2.2) that for all $\sigma \in \mathfrak{S}_{n}$,

$$
F_{\operatorname{EXD}(\sigma), n}\left(1, q, q^{2}, \ldots\right)=\frac{q^{\operatorname{maj}(\sigma)-\operatorname{exc}(\sigma)}}{(1-q)\left(1-q^{2}\right) \ldots\left(1-q^{n}\right)} .
$$

For any $n, j, k \geq 0$, let

$$
Q_{n, j, k}=Q_{n, j, k}(\mathbf{x}):=\sum_{\begin{array}{c}
\sigma \in \mathfrak{S}_{n} \\
\operatorname{exc}(\sigma)=j \\
\operatorname{fix}(\sigma)=k
\end{array}} F_{\operatorname{EXD}(\sigma), n}(\mathbf{x})
$$

By taking the specialization of the generating function, we get

$$
\left.\sum_{n, j, k \geq 0} Q_{n, j, k}(\mathbf{x}) t^{j} r^{k} z^{n}\right|_{\substack{x_{i}:=q^{i-1} \\ z:=z(1-q)}}=\sum_{n \geq 0} \sum_{\sigma \in \mathfrak{S}_{n}} q^{\operatorname{maj}(\sigma)-\operatorname{exc}(\sigma)} t^{\operatorname{exc}(\sigma)} r^{\mathrm{fix}(\sigma)} \frac{z^{n}}{[n]_{q} !} .
$$

It follows from (2.1) and (2.3) that by setting $x_{i}:=q^{i-1}, z:=z(1-q)$ and $t:=t q$ in the following result we obtain Theorem 1.2 .

\section{Theorem 2.1.}

$$
\sum_{n, j, k \geq 0} Q_{n, j, k} t^{j} r^{k} z^{n}=\frac{(1-t) H(z r)}{H(z t)-t H(z)} .
$$

The proof of this theorem requires an alternative characterization of $Q_{n, j, k}$, which is established by adapting a bijection that Gessel and Reutenauer [19] introduced to enumerate permutations with a fixed descent set and a fixed cycle type. Gessel and Reutenauer deal with circular words over the alphabet of positive integers. We consider circular words over the alphabet of barred and unbarred positive integers. For each such circular word and each starting position, one gets a linear word by reading the circular word in a clockwise direction. If one gets a distinct linear word for each starting position, then the circular word is said to be primitive. For example, $(\overline{1}, 1,1)$ is primitive while $(\overline{1}, 2, \overline{1}, 2)$ is not. The absolute value of a letter is the letter obtained by erasing the bar if there is one. We will say that a primitive circular word is a necklace if each letter that is followed (clockwise) by a letter greater in absolute value is barred and each letter that is followed by a letter smaller in absolute value is unbarred. Letters that are followed by letters equal in 
absolute value have the option of being barred or not. A circular word consisting of one barred letter is not a necklace. For example the following circular words are necklaces:

$$
(\overline{1}, 3,1, \overline{1}, 2,2),(\overline{1}, 3, \overline{1}, \overline{1}, 2,2),(\overline{1}, 3,1, \overline{1}, \overline{2}, 2),(\overline{1}, 3, \overline{1}, \overline{1}, \overline{2}, 2),(3),
$$

while $(\overline{1}, \overline{3}, 1,1,2, \overline{2})$ and $(\overline{3})$ are not.

An ornament is a multiset of necklaces. The type $\lambda(R)$ of an ornament $R$ is the partition whose parts are the sizes of the necklaces in $R$. The weight $w(R)$ of an ornament $R$ is the product of the weights of the letters of $R$, where the weight of the letter $a$ is the indeterminate $x_{|a|}$, where $|a|$ denotes the absolute value of $a$. For example,

$$
\lambda((\overline{1}, 2,2),(\overline{1}, \overline{2}, 3,3,2))=(5,3)
$$

and

$$
w((\overline{1}, 2,2),(\overline{1}, \overline{2}, 3,3,2))=x_{1}^{2} x_{2}^{4} x_{3}^{2} .
$$

For each partition $\lambda$ and nonnegative integer $j$, let $\mathfrak{R}_{\lambda, j}$ be the set of ornaments of type $\lambda$ with $j$ bars.

Theorem 2.2. For all $\lambda \vdash n$ and $j=0,1, \ldots, n-1$, let

$$
Q_{\lambda, j}:=\sum_{\sigma} F_{\operatorname{EXD}(\sigma), n}
$$

summed over all permutations of cycle type $\lambda$ with $j$ excedances. Then

$$
Q_{\lambda, j}=\sum_{R \in \Re_{\lambda, j}} w(R) .
$$

This theorem is proved via a bijection between ornaments of type $\lambda$ with $j$ bars and permutations of cycle type $\lambda$ with $j$ excedances paired with "compatible" weakly decreasing sequences of positive integers. Our bijection is an adaptation of the Gessel-Reutenauer bijection, which sends multisets of primitive circular words over an ordered alphabet to permutations paired with compatible weakly decreasing sequences over the same alphabet. The Gessel-Reutenauer bijection, which is also described in 8, can be viewed as a necklace analog of the bijection in Stanley's theory of P-partitions [30. Here we need to order our alphabet by

$$
1<\overline{1}<2<\overline{2}<\cdots .
$$

Our map is obtained by first applying the Gessel-Reutenauer map to our ornaments and then removing the bars from the barred letters in the compatible weakly decreasing sequence to obtain a weakly decreasing sequence of positive integers.

Theorem 2.2 has several interesting consequences. For one thing, it can be used to prove that the quasisymmetric functions $Q_{\lambda, j}$ and $Q_{n, j, k}$ are actually symmetric. It also has the following useful consequence.

Corollary 2.3. For all $n, j, k$,

$$
Q_{n, j, k}=h_{k} Q_{n-k, j, 0} .
$$

It follows from Corollary 2.3 that Theorem 2.1 is equivalent to

$$
\sum_{n, j \geq 0} Q_{n, j, 0} t^{j} z^{n}=\frac{1-t}{H(z t)-t H(z)}
$$


which in turn is equivalent to the recurrence relation

$$
Q_{n, j, 0}=\sum_{\substack{0 \leq m \leq n-2 \\ j+m-n<i<j}} Q_{m, i, 0} h_{n-m} .
$$

We establish this recurrence relation by introducing another type of configuration, closely related to ornaments.

Define a banner $B$ to be a word over the alphabet of barred and unbarred positive integers, where $B(i)$ is barred if $|B(i)|<|B(i+1)|$, and $B(i)$ is unbarred if $|B(i)|>$ $|B(i+1)|$ or $i=\operatorname{length}(B)$. All other letters have the option of being barred. The weight of a banner is the product of the weights of its letters.

A Lyndon word over an ordered alphabet is a word that is strictly lexicographically smaller than all its circular rearrangements. A Lyndon factorization of a word over an ordered alphabet is a factorization into a weakly lexicographically decreasing sequence of Lyndon words. It is a result of Lyndon (see [24, Theorem 5.1.5]) that every word has a unique Lyndon factorization. The Lyndon type of a word is the partition whose parts are the lengths of the words in its Lyndon factorization. For each partition $\lambda$ and positive integer $j$, let $\mathfrak{B}_{\lambda, j}$ be the set of banners with $j$ bars whose Lyndon type is $\lambda$.

By turning the Lyndon words in the Lyndon factorization of a banner into circular words, we obtain an ornament. This map from banners to ornaments is the bijection whose existence is asserted in the following proposition.

Proposition 2.4. For any partition $\lambda$ and nonnegative integer $j$, there is a weightpreserving bijection from $\mathfrak{B}_{\lambda, j}$ to $\mathfrak{R}_{\lambda, j}$.

Corollary 2.5. Let $\mathfrak{B}_{n, j}$ be the set of banners of length $n$ with $j$ bars whose Lyndon type has no parts of size 1 . Then

$$
Q_{n, j, 0}=\sum_{B \in \mathfrak{B}_{n, j}} w(B) .
$$

Define a marked sequence $(\alpha, i)$ to be a weakly increasing finite sequence $\alpha$ of positive integers together with an integer $i$ such that $1 \leq i \leq \operatorname{length}(\alpha)-1$. Let $\mathfrak{M}_{n}$ be the set of marked sequences of length $n$ and let $\mathfrak{B}_{n}$ be the set of banners of length $n$ whose Lyndon type has no parts of size 1.

Theorem 2.6. For all $n \geq 2$, there is a bijection

$$
\gamma: \mathfrak{B}_{n} \rightarrow \bigcup_{0 \leq m \leq n-2} \mathfrak{B}_{m} \times \mathfrak{M}_{n-m}
$$

such that if $\gamma(B)=\left(B^{\prime},(\alpha, i)\right)$ then

$$
w(B)=w\left(B^{\prime}\right) w(\alpha)
$$

and

$$
\operatorname{bar}(B)=\operatorname{bar}\left(B^{\prime}\right)+i,
$$

where $\operatorname{bar}(B)$ denotes the number of bars of $B$.

We will not describe the bijection here except for saying that, when restricted to banners with distinct letters, it reduces to a bijection from permutations to marked words that Stembridge [35] constructed to study the representation of the symmetric group on the cohomology of the toric variety assoiciated with the type A Coxeter complex. (We discuss this representation in Section 3) Banners in 
$\mathfrak{B}_{n}$ admit a certain kind of decomposition, called a decreasing decomposition in [8]. The decreasing decomposition plays the role in our bijection that the cycle decomposition of permutations plays in Stembridge's bijection.

Corollary 2.5 and Theorem 2.6 are all that is needed to establish the recurrence relation (2.6), which yields our main result, Theorem 2.1.

Remark. If one applies the standard involution $\omega$ to the symmetric function appearing on the right-hand side of (2.4), one gets a refinement of a symmetric function that has been studied by Carlitz, Scoville, and Vaughan [6] and Dollhopf, Goulden, and Greene [10] in connection with the enumeration of words with no adjacent repeats. It was pointed out to us by Richard Stanley that these words and our banners can be viewed as "dual" graph colorings in the sense of [34, Theorem 4.2].

\section{Some RePRESENTATION-THEORETIC CONSEQUENCES}

The Frobenius characteristic ch is a fundamental homomorphism from the ring of representations of symmetric groups to the ring of symmetric functions. In this section we present two representations whose Frobenius characteristic is $Q_{n, j}:=$ $\sum_{k=0}^{n} Q_{n, j, k}$.

The first representation involves the toric variety associated with the Coxeter complex of a Weyl group. Let $X_{n}$ be the toric variety associated with the Coxeter complex of $\mathfrak{S}_{n}$. The action of $\mathfrak{S}_{n}$ on $X_{n}$ induces a representation of $\mathfrak{S}_{n}$ on the cohomology $H^{2 j}\left(X_{n}\right)$ for each $j=0, \ldots, n-1$. (Cohomology in odd degree vanishes.) Stanley [32, using a formula of Processi [25, proves that

$$
\sum_{n \geq 0} \sum_{j=0}^{n-1} \operatorname{ch} H^{2 j}\left(X_{n}\right) t^{j} z^{n}=\frac{(1-t) H(z)}{H(z t)-t H(z)} .
$$

Combining this with Theorem 2.1 yields the following conclusion.

Theorem 3.1. For all $j=0,1, \ldots, n-1$,

$$
\operatorname{ch} H^{2 j}\left(X_{n}\right)=Q_{n, j} .
$$

The second representation involves poset topology, a subject in which topological properties of a simplicial complex associated with a poset are studied; see [37. The faces of the simplicial complex, called the order complex of the poset, are the chains of the poset. Here we consider the homology of the order complex of the Rees product of two simple posets. The Rees product is a poset construction recently introduced by Björner and Welker [4] in their study of relations between poset topology and commutative algebra.

Definition 3.2. Let $P$ and $Q$ be pure (ranked) posets with respective rank functions $r_{P}$ and $r_{Q}$. The Rees product $P * Q$ of $P$ and $Q$ is defined as follows:

$$
P * Q:=\left\{(p, q) \in P \times Q: r_{P}(p) \geq r_{Q}(q)\right\}
$$

with order relation given by $\left(p_{1}, q_{1}\right) \leq\left(p_{2}, q_{2}\right)$ if the following holds:

- $p_{1} \leq_{P} p_{2}$,

- $q_{1} \leq_{Q} q_{2}$,

- $r_{P}\left(p_{2}\right)-r_{P}\left(p_{1}\right) \geq r_{Q}\left(q_{2}\right)-r_{Q}\left(q_{1}\right)$. 
Let $B_{n}$ be the Boolean algebra (i.e., the lattice of subsets of $[n]$ ordered by inclusion) and let $C_{n}$ be the chain $1<2<\cdots<n$. The maximal elements of $\left(B_{n} \backslash\{\emptyset\}\right) * C_{n}$ are of the form $([n], j)$, where $j=1, \ldots, n$. Let $I_{n, j}$ be the set of elements of $\left(B_{n} \backslash\{\emptyset\}\right) * C_{n}$ that are smaller than $([n], j)$, and let $\tilde{H}_{i}\left(I_{n, j}\right)$ be the reduced simplicial (complex) homology of the order complex of $I_{n, j}$. It follows from results of Björner and Welker that homology vanishes below the top dimension $n-2$. The symmetric group $\mathfrak{S}_{n}$ acts on $I_{n, j}$ in an obvious way, and this induces a representation on $\tilde{H}_{n-2}\left(I_{n, j}\right)$. We prove the following result using techniques from poset topology.

\section{Theorem 3.3.}

$$
1+\sum_{n \geq 1} \sum_{j=1}^{n} \operatorname{ch}\left(\tilde{H}_{n-2}\left(I_{n, j}\right) \otimes \operatorname{sgn}\right) t^{j-1} z^{n}=\frac{(1-t) H(z)}{H(z t)-t H(z)},
$$

where sgn denotes the sign representation. Consequently, for all $n, j$,

$$
\operatorname{ch}\left(\tilde{H}_{n-2}\left(I_{n, j}\right) \otimes \operatorname{sgn}\right)=Q_{n, j-1}
$$

and as $\mathfrak{S}_{n}$-modules

$$
\tilde{H}_{n-2}\left(I_{n, j}\right) \otimes \operatorname{sgn} \cong H^{2 j-2}\left(X_{n}\right) .
$$

We conjecture that for all $\lambda$ and $j$, the symmetric function $Q_{\lambda, j}$ is also the Frobenius characteristic of some representation. One consequence of Theorem 2.2 is that $Q_{\lambda, j}$ can be described as a product of plethysms of symmetric functions of the form $Q_{(n), i}$, where $(n)$ denotes a partition with a single part. Hence if the conjecture holds for all $Q_{(n), i}$, then it holds in general. We use ornaments and banners to show that if the conjecture does hold, then the restriction to $\mathfrak{S}_{n-1}$ of the representation whose Frobenius characteristic is $Q_{(n), i}$, has Frobenius characteristic $Q_{n-1, i-1}$.

\section{A new Mahonian statistic}

In this section we describe a new Mahonian statistic whose joint distribution with des is the same as the joint distribution of maj and exc.

An admissible inversion of $\sigma \in \mathfrak{S}_{n}$ is a pair $(\sigma(i), \sigma(j))$ such that the following conditions hold:

- $i<j$,

- $\sigma(i)>\sigma(j)$,

- either

○ $\sigma(j)<\sigma(j+1)$ or

$\circ \exists k$ such that $i<k<j$ and $\sigma(k)<\sigma(j)$.

Let ai $(\sigma):=\#$ admissible inversions of $\sigma$. Define the statistic

$$
\operatorname{aid}(\sigma):=\operatorname{ai}(\sigma)+\operatorname{des}(\sigma) \text {. }
$$

For example, the admissible inversions of 24153 are $(2,1),(4,1)$ and $(4,3)$. So $\operatorname{aid}(24153)=3+2$.

Theorem 4.1. For all $n \geq 1$,

$$
\sum_{\sigma \in \mathfrak{S}_{n}} q^{\operatorname{aid}(\sigma)} t^{\operatorname{des}(\sigma)}=\sum_{\sigma \in \mathfrak{S}_{n}} q^{\operatorname{maj}(\sigma)} t^{\operatorname{exc}(\sigma)} .
$$


We do not have a direct proof of this simple identity except when $t$ or $q$ is 1 . Our proof relies on Theorem 1.1, a $q$-analog of Theorem [3.3, and techniques from poset topology. We consider the Rees product $\left(B_{n}(q) \backslash\{(0)\}\right) * C_{n}$, where $B_{n}(q)$ is the lattice of subspaces of the vector space $\mathbb{F}_{q}^{n}$. Let $I_{n, j}(q)$ be the set of elements in $\left(B_{n}(q) \backslash\{(0)\}\right) * C_{n}$ that are less than the maximal element $\left(\mathbb{F}_{q}^{n}, j\right)$. We first use a well-known tool from poset topology, called lexicographic shellability [3, 37, to prove that

$$
\operatorname{dim} \tilde{H}_{n-2}\left(I_{n, j}(q)\right)=\sum_{\substack{\sigma \in \mathfrak{S}_{n} \\ \operatorname{des}(\sigma)=j-1}} q^{\mathrm{ai}(\sigma)} .
$$

We then use other tools from poset topology to prove a theorem analogous to Theorem 3.3, which states that

$$
\sum_{n \geq 0} \sum_{j=1}^{n} \operatorname{dim} \tilde{H}_{n-2}\left(I_{n, j}(q)\right) t^{j-1} \frac{z^{n}}{[n]_{q} !}=\frac{(1-t) \exp _{q}(z)}{\exp _{q}(z t)-t \exp _{q}(z)}
$$

Theorem 4.1 now follows from Theorem 1.1 and equation (4.1).

\section{Acknowledgements}

The research presented here began while both authors were visiting the MittagLeffler Institute as participants in a combinatorics program organized by Anders Björner and Richard Stanley. We thank the Institute for its hospitality and support. We are also grateful to Ira Gessel for some very useful discussions and to Dominique Foata for valuable comments, which included (1.4) and an improved formulation of Theorem 1.2,

\section{REFERENCES}

[1] E. Babson and E. Steingrímsson, Generalized permutation patterns and a classification of the Mahonian statistics, Sém. Lothar. Combin., B44b (2000), 18 pp. MR.1758852 (2002b:05006)

[2] D. Beck and J.B. Remmel, Permutation enumeration of the symmetric group and the combinatorics of symmetric functions, J. Combin. Theory Ser. A 72 (1995), 1-49. MR1354966 (96j:05113)

[3] A. Björner, Shellable and Cohen-Macaulay partially ordered sets, Trans. AMS 260 (1980), 159-183. MR.570784 (81i:06001)

[4] A. Björner and V. Welker, Segre and Rees products of posets, with ring-theoretic applications, J. Pure Appl. Algebra 198 (2005), 43-55. MR2132872 (2005m:06006)

[5] L. Carlitz, A combinatorial property of q-Eulerian numbers, The American Mathematical Monthly 82 (1975), 51-54. MR0366683 (51:2930)

[6] L. Carlitz, R. Scoville, and T. Vaughan, Enumeration of pairs of sequences by rises, falls and levels, Manuscripta Math. 19 (1976), 211-243. MR0432472 (55:5460)

[7] R.J. Clarke, E. Steingrímsson, and J. Zeng, New Euler-Mahonian statistics on permutations and words, Adv. in Appl. Math. 18 (1997), 237-270. MR1436481 (97m:05008)

[8] J. Désarménien and M.L. Wachs, Descent classes of permutations with a given number of fixed points, J. Combin. Theory Ser. A 64 (1993), no. 2, 311-328. MR.1245164 (95c:05008)

[9] I. Dolgachev and V. Lunts, A character formula for the representation of a Weyl group in the cohomology of the associated toric variety, J. Algebra 168 (1994), 741-772. MR1293622 (95h:20008)

[10] J. Dollhopf, I. Goulden, and C. Greene, Words avoiding a reflexive acyclic relation, Electon. J. Combin. 11 (2006) \#R28. MR 2224941|(2007b:05013)

[11] D. Foata, Sur un énoncé de MacMahon, C. R. Acad. Sci. Paris 258 (1964), 1672-1675. MR0158834 (28:2057) 
[12] D. Foata, On the Netto inversion number of a sequence, Proc. Amer. Math. Soc. 19 (1968), 236-240. MR0223256 (36:6304)

[13] D. Foata, Distributions eulériennes et mahoniennes sur le groupe des permutations, NATO Adv. Study Inst. Ser., Ser. C: Math. Phys. Sci., 31, Higher combinatorics (Proc. NATO Advanced Study Inst., Berlin, 1976), pp. 27-49, Reidel, Dordrecht-Boston, Mass., 1977. MR519777 (80e:05011)

[14] D. Foata, Rearrangements of words, in M. Lothaire, Combinatorics on Words, Ch. 10, Encyclopedia of Math. and its Appl., Vol. 17, Addison-Wesley, Reading, MA, 1983. MR675953 (84g:05002)

[15] D. Foata and M.-P. Schützenberger, Théorie géométrique des polynômes eulériens, Lecture Notes in Mathematics, Vol. 138 Springer-Verlag, Berlin-New York, 1970. MR0272642 $(42: 7523)$

[16] D. Foata and M.-P. Schützenberger, Major index and inversion number of permutations, Math. Nachr. 83 (1978), 143-159. MR506852 (81d:05007)

[17] D. Foata and D. Zeilberger, Denert's permutation statistic is indeed Euler-Mahonian, Stud. Appl. Math. 83 (1990), 31-59. MR.1061147 (91h:05016)

[18] A.M. Garsia and I. Gessel, Permutation statistics and partitions, Adv. in Math. 31 (1979), 288-305. MR532836 (80e:05013)

[19] I.M. Gessel and C. Reutenauer, Counting permutations with given cycle structure and descent set, J. Combin. Theory Ser. A 64 (1993), 189-215. MR1245159 (95g:05006)

[20] J. Haglund, q-Rook polynomials and matrices over finite fields, Adv. in Appl. Math. 20 (1998), 450-487. MR:1612854 (99f:05004)

[21] D. Knuth, The Art of Computer Programming, Vol. 3. Sorting and Searching, Second Edition, Addison-Wesley, Reading, MA, 1998. MR0445948 (56:4281)

[22] P.A. MacMahon, Combinatory Analysis, 2 volumes, Cambridge University Press, London, 1915-1916. Reprinted by Chelsea, New York, 1960. MR0141605 (25:5003)

[23] P.A. MacMahon, The indices of permutations and the derivation therefrom of functions of a single variable associated with the permutations of any assemblage of objects, Amer. J. Math. 35 (1913), no. 3, 281-322. MR1506186

[24] D. Perrin, Factorizations of Free Monoids, in M. Lothaire, Combinatorics on Words, Ch. 5, Encyclopedia of Math. and its Appl., Vol. 17, Addison-Wesley, Reading, MA, 1983. MR.675953 (84g:05002)

[25] C. Procesi, The toric variety associated to Weyl chambers, Mots, 153-161, Lang. Raison. Calc., Hermès, Paris, 1990. MR.1252661 (94k:14045)

[26] A. Ram, J. Remmel, and T. Whitehead, Combinatorics of the q-basis of symmetric functions, J. Combin. Theory Ser. A 76 (1996), 231-271. MR.1416016 (98g:05151)

[27] D. Rawlings, Enumeration of permutations by descents, idescents, imajor index, and basic components, J. Combin. Theory Ser. A 36 (1984), 1-14. MR728499 (85k:05013)

[28] O. Rodrigues, Note sur les inversions, ou derangements produits dans les permutations, Journal de Mathematiques 4 (1839), 236-240.

[29] M. Skandera, An Eulerian partner for inversions, Sém. Lothar. Combin. 46 (2001/02), Art. B46d, 19 pp. (electronic). MR.1848722 (2002g:05010)

[30] R.P. Stanley, Ordered structures and partitions, Memoirs Amer. Math. Soc. 119 (1972). MR0332509 (48:10836)

[31] R.P. Stanley, Binomial posets, Möbius inversion, and permutation enumeration, J. Combinatorial Theory Ser. A 20 (1976), 336-356. MR0409206 (53:12968)

[32] R.P. Stanley, Log-concave and unimodal sequences in algebra, combinatorics, and geometry, Graph theory and its applications: East and West (Jinan, 1986), 500-535, Ann. New York Acad. Sci., 576, New York Acad. Sci., New York, 1989. MR.1110850 (92e:05124)

[33] R.P. Stanley, Enumerative combinatorics. Vol. 2. Cambridge Studies in Advanced Mathematics, 62. Cambridge University Press, Cambridge, 1999. MR.1676282 (2000k:05026)

[34] R.P. Stanley, A symmetric function generalization of the chromatic polynomial of a graph, Advances in Math. 111 (1995), 166-194. MR1317387 (96b:05174)

[35] J.R. Stembridge, Eulerian numbers, tableaux, and the Betti numbers of a toric variety, Discrete Math. 99 (1992), 307-320. MR.1158793 (93f:05103)

[36] J.R. Stembridge, Some permutation representations of Weyl groups associated with the cohomology of toric varieties, Adv. Math. 106 (1994), 244-301. MR1279220 (95f:20011) 
$q$-EULERIAN POLYNOMIALS: EXCEDANCE NUMBER AND MAJOR INDEX

[37] M.L. Wachs, Poset topology: tools and applications, to appear as chapter of Geometric Combinatorics volume of PCMI lecture notes series. ArXiv math.CO/0602226.

[38] M.L. Wachs, An involution for signed Eulerian numbers, Discrete Math. 99 (1992), 59-62. MR.1158780 (93e:11030b)

Department of Mathematics, Washington University, St. Louis, Missouri 63130

E-mail address: shareshi@math.wustl.edu

Department of Mathematics, University of Miami, Coral Gables, Florida 33124

E-mail address: wachs@math.miami.edu 